\title{
CONFÉRENCE
}

\section{Pathologies infectieuses des maxillaires et tomographie volumique par faisceau cônique}

\section{Claude Hodez}

L'infection osseuse est classiquement dénommée ostéite lorsqu'elle se développe localement, et ostéomyélite quand elle est le fait d'une greffe microbienne par voie hématogène.

Avant la survenue de l'imagerie en coupes, la radiologie dento-maxillaire s'attachait à la recherche des infections dentaires avec leurs retentissements juxta radiculaires et apicaux.

Le scanner d'abord mais surtout l'imagerie CBCT ont complété cette approche en permettant la mise en évidence d'extensions intra osseuses qui restaient jusqu'alors insoupçonnées.

Que l'infection soit focale ou extensive, qu'elle évolue sur un mode aigu, subaigu ou chronique, il existe une séméiologie radiologique qu'il convient de connaitre car les ostéites sont plus fréquentes qu'on ne l'imagine. Leur méconnaissance peut mener à des tableaux graves avec des retards de diagnostic portant parfois sur plusieurs mois. Dans les formes les plus évoluées, la durée du traitement peut se compter également en mois ou en années.

Quand la clinique évoque la possibilité d'une ostéite, l'imagerie classique de projection est inopérante et ne peut mener qu'à des faux négatifs et des retards de diagnostic. Seul l'examen $\mathrm{CBCT}$ en haute résolution effectué par un opérateur expérimenté peut permettre le diagnostic. Les signes de début très ténus peuvent passer complètement inaperçus sans un examen attentif et orienté.

L'agression septique de l'os associe des phénomènes de destruction et de reconstruction dans des proportions très variables. Classiquement la destruction caractérise plutôt les phases aiguës et subaiguës alors que la reconstruction, source d'ostéosclérose, se voit dans les atteintes chroniques ou les phases de cicatrisation, mais tous les modes évolutifs sont possibles.

Sur les coupes en haute résolution on s'attachera à rechercher les premiers signes de l'infection osseuse :

- Irrégularité du liseré de reconstruction osseuse dans une cavité d'avulsion

- Petites zones de déminéralisation affectant l'os trabéculé et/ou l'os compact.

- Trajets fistuleux prenant naissance dans la zone d'infection initiale et s'étendant dans l'os trabéculé parfois loin à distance.

- Trajets fistuleux très fins traversant l'os cortical.

- Déminéralisation progressive de l'os cortical dont la densité se rapproche de celle de l'os trabéculé : c'est la dédifférenciation cortico-trabéculée

- Apparition en bordure de l'os cortical et en regard des trajets fistuleux de réactions de reconstruction osseuse de la part du périoste. Ce sont les appositions périostées lamellaires ou en pelure d'oignon.

This is an Open Access article distributed under the terms of the Creative Commons Attribution License 4.0, which permits unrestricted use, distribution, and reproduction in any medium, provided the original work is properly cited. 
- Aspect « dépoli », irrégulier, granité de la surface osseuse quand on examine en imagerie 3D les reconstructions dites " de surface".

- Fragments osseux nécrotiques denses (séquestres).

Les ostéites mandibulaires semblent plus fréquentes et plus graves que les localisations maxillaires, sans doute pour des raisons de mode de vascularisation mais l'imagerie fine révèle souvent dans les maxillaires des plages déminéralisées ou de trajets fistuleux au voisinage de foyers dentaires ou de poches parodontales susceptibles d'être à l'origine de sinusites.

L'ostéonécrose des maxillaires peut donner un tableau trompeur d'ostéite mais le contexte de traitement par biphosphonates doit permettre de redresser le diagnostic. 